\title{
Triterpenoids from Momordica balsamina with a Collateral Sensitivity Effect for Tackling Multidrug Resistance in Cancer Cells
}

\author{
Authors \\ Affiliations \\ 1 Research Institute for Medicines (iMed.ULisboa), Faculty of \\ Pharmacy, Universidade de Lisboa, Lisbon, Portugal \\ 2 ATLÂNTICA - Escola Universitária de Ciências Empresariais, \\ Saúde, Tecnologias e Engenharia, Oeiras, Portugal \\ 3 Centro de Estudos Moçambicanos e de Etnociências, \\ Faculty of Natural Sciences and Mathematics, Pedagogical \\ University, Maputo, Mozambique \\ 4 Institute of Pathology, University Hospital Charité, \\ Berlin, Germany \\ 5 Department of Pathology, Vivantes Clinics, Berlin, \\ Germany
}

Cátia Ramalhete $^{1,2}$, Silva Mulhovo ${ }^{3}$, Hermann Lage ${ }^{4,5} \uparrow$, Maria-José U. Ferreira ${ }^{1}$

Key words

Momordica balsamina, Cucurbitaceae, collateral sensitivity, antiproliferative activity, multidrug resistance, triterpenes

$\begin{array}{ll}\text { received } & \text { March 27, } 2018 \\ \text { revised } & \text { June } 5,2018 \\ \text { accepted } & \text { June 22, } 2018\end{array}$

Bibliography

DOI https://doi.org/10.1055/a-0651-8141

Published online July 11, 2018 | Planta Med 2018; 84: 1372-

1379 @ Georg Thieme Verlag KG Stuttgart · New York I

ISSN 0032-0943

Correspondence

Prof. Dr. Maria José U. Ferreira

Research Institute for Medicines (iMed.ULisboa), Faculty of

Pharmacy, Universidade de Lisboa

Av. Prof. Gama Pinto, 1649-003 Lisbon, Portugal

Phone: + 351217946475 , Fax: + 351217946470

mjuferreira@ff.ulisboa.pt
Supporting information available online at

http://www.thieme-connect.de/products

\section{ABSTRACT}

The collateral sensitivity effect is among the most promising strategies for overcoming multidrug resistance in cancer. In this work, 28 cucurbitane-type triterpenoids (1-28), previously isolated from the African medicinal plant Momordica balsamina and its derivatives, were evaluated for their collateral sensitivity effect on three different human cancer entities, gastric (EPG85-257), pancreatic (EPP85-181), and colon (HT29), each with two different multidrug-resistant variants. One was selected for its resistance to daunorubicin (EPG85257RDB, EPP85-181RDB, HT-29RDB) and the other was selected for its resistance to mitoxantrone (EPG85-257RNOV, EPP85-181RNOV, HT-29RNOV). On gastric cell lines, the best results were obtained for compounds 3 and 10 , which exhibited a collateral sensitivity effect together with high antiproliferative activity. In turn, on colon cancer cell lines, the best multidrug resistance-selective antiproliferative effects were observed for derivatives 11,13 , and 15 , which showed collateral sensitivity effects against both resistant variants. Compounds 11 and 3 were also the most selective against the multidrug resistance pancreatic cells lines. Some compounds, such $6,10,11$ and 15, were previously found to be strong Pglycoprotein modulators, thus highlighting their potential as promising leads for overcoming multidrug resistance in cancer cells.

\section{Introduction}

In spite of all the valuable chemotherapy regimens to treat cancer, it represents a major health problem worldwide, especially due to the high incidence of MDR phenotypes. MDR is characterized by cross-resistance of tumors to multiple structurally and functionally unrelated drugs. One of the most relevant and studied MDR mechanisms of tumor cells is correlated with the overexpression of P-gp (P-gp/ABCB1/MDR1), encoded by the $A B C B 1$ gene, which belongs to the superfamily of $A B C$ transporters [1]. The overexpression of this $A B C$ transporter, resulting from an association of intrinsic and acquired drug resistance factors, is evident in tumor tissues from patients, reducing the intracellular accumulation of the anticancer drug and thus compromising the efficacy of treatment [2]. As a consequence of the efflux function of P-gp, clinical chemotherapeutic agents, such as paclitaxel and Adriamycin, or the selective kinase inhibitors erlotinib and sorafenib suffered a reduction of efficacy [3-5].

Numerous strategies to overcome MDR have been explored, including the development of P-gp modulators to restore drug accumulation, the design of novel drugs that avoid recognition and efflux, and the use of small molecules that selectively kill MDR 


\section{ABBREVIATIONS \\ ABC ATP-binding cassette \\ ABCB1 ATP-binding cassette, subfamily B \\ $\mathrm{Cl} \quad$ confidence interval \\ EPG85-257P parental gastric cancer cells \\ EPG85-257RDB gastric cancer cells selected against daunorubicin \\ EPG85-257RNOV gastric cancer cells selected against mitoxantrone \\ EPP85-181RDB pancreatic cancer cells selected against daunorubicin \\ EPP85-181P parental pancreatic cancer cells \\ EPP85-181RNOV pancreatic cancer cells selected against mitoxantrone \\ MDR multidrug resistance \\ MDR1 multidrug resistance gene 1 \\ P-gp P-glycoprotein \\ RDB daunorubicin \\ RNOV mitoxantrone \\ RR relative resistance \\ SRB sulforhodamine B}

chemical mechanisms, in relation to MRP1-overexpressing cancer cells, there is experimental evidence that they mainly act as stimulators of MRP1-mediated glutathione efflux, thus modifying redox balance, which selectively triggers apoptosis of resistant cells overexpressing this $A B C$ protein [7].

Momordica balsmamina L. (Cucurbitaceae), commonly called African pumpkin, is an herb commonly found in tropical and subtropical regions of Africa and Asia. It presents high nutritional and medicinal value, being extensively used as food and traditional medicine [9]. A wide variety of cucurbitane-type triterpenoids with different biological activities has been isolated from the Momordica genus, namely from Momordica charantia [10,11].

In our previous investigation of the methanol extract of the aerial parts of $M$. balsamina, several cucurbitanes isolated or obtained by derivatization showed they were potent inhibitors of P-gp efflux pump activity $[12,13]$. Moreover, they were also able to inhibit the efflux pump systems of resistant strains of grampositive bacteria [14].

In the present work and continuing our search for plant-derived compounds that can circumvent MDR [15-20], triterpenoids 1-28 were evaluated for their potential collateral sensitivity effect on colon, gastric, and cancer cell models (drug sensitive and drug resistant sublines) well characterized for MDR [17].

\section{Results and Discussion}

The phytochemical study of the methanol extract of the aerial plant parts of $M$. balsamina lead to the isolation of several triterpenes (1-10) ( $\triangleright$ Fig. 1) with the cucurbitane skeleton, as previously described $[12,21-23]$. Karavilagenin C [10,7 $\beta$-methoxycucurbita-5,24-diene-3 $\beta, 23(R)$-diol], the major compound, allowed for the generation of a small library of mono- and di-acyl derivatives at C-23 and/or C-3, bearing alkanoyl, aroyl, and cinnamoyl moieties (11-28) ( $\bullet$ Fig. 1) [12, 24]. These compounds were previously evaluated at non-cytotoxic doses for their ability as P-glycoprotein modulators on MDR1 mouse lymphoma cells [12,13]. It was concluded that different substitution patterns, at both the tetracyclic nucleus and the side chain, led to distinct inhibition of this efflux pump activity $[12,13]$.

In this work, aiming at finding effective compounds for overcoming MDR, compounds 1-28 were assessed for their potential collateral sensitivity effect on three different cancer cell models: gastric (EPG85-257), pancreatic (EPP85-181), and colon (HT-29) cancer cells. For each cancer cell model, one sensitive cell line and two resistant sublines, selected for resistance to RDB and to RNOV, were tested. The characteristics of these MDR cell lines are well known and the same cancer cell models were used with a similar purpose in other studies [15-17,25-27] The collateral sensitivity effect was assessed by determining the RR (calculated as the ratio of the $\mathrm{IC}_{50}$ of a compound against a resistant line divided by the $I C_{50}$ against the corresponding parental line). Compounds with an $R R<1$ kill MDR cells more effectively than parental cells, and when they exhibit an RR $\leq 0.50$ they have a collateral sensitivity effect. An RR $\geq 2.0$ expresses a compound that has resistance to a drug and is simultaneously cross-resistance to others [28]. The RR ratio only evaluates selectivity towards resistant cells. Thus, when selecting a compound with a collateral sen- 


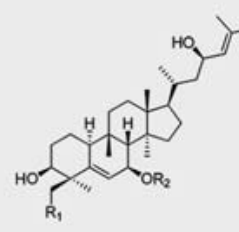

1: $R_{1}=O H ; R_{2}=H$

10. $\mathrm{R}_{1}=\mathrm{H}_{2} \mathrm{R}_{2}=\mathrm{CH}_{3}$
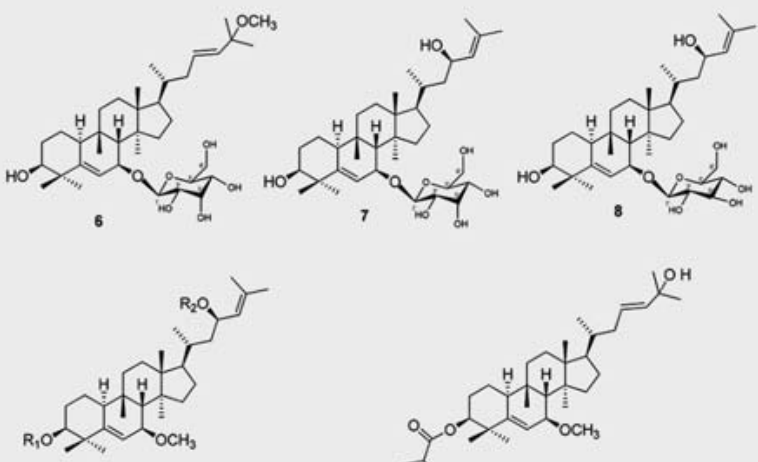

11: $R_{1}=H ; R_{2}=\mathrm{COCH}_{3}$

12: $R_{1}=R_{2}=\mathrm{COCH}_{3}$

13: $\mathrm{R}_{1}=\mathrm{H}_{;} \mathrm{R}_{2}=\mathrm{COCH}_{2} \mathrm{CH}_{3}$

14: $\mathrm{R}_{1}=\mathrm{R}_{2}=\mathrm{COCH}_{2} \mathrm{CH}_{3}$
15: $\mathrm{R}_{1}=\mathrm{H}: \mathrm{R}_{2}=\mathrm{COCH}_{2} \mathrm{CH}_{2} \mathrm{CH}_{3}$
16: $\mathrm{R}_{1}=\mathrm{R}_{2}=\mathrm{COCH}_{2} \mathrm{CH}_{2} \mathrm{CH}_{3}$

18: $R_{1}=R_{2}=B z$

19: $R_{1}=H ; R_{2}=\rho$-nitroBz

20: $R_{1}=R_{2}=p$-nitroBz

21: $R_{1}=H ; R_{2}=p$-chloroB

22: $R_{1}=R_{2}=p$-chloroBz

23: $R_{1}=H, R_{2}=\rho$-methoxy $B_{2}$

24: $R_{1}=R_{2}=p$-methoxy $8 z$

25: $R_{1}=H ; R_{2}=C$ in

26: $R_{1}=R_{2}=$ Cin

27: $\mathrm{R}_{1}=\mathrm{H} ; \mathrm{R}_{2}=\mathrm{COCH}_{2} \mathrm{CH}_{2} \mathrm{COO}$

28: $R_{1}=R_{2}=\mathrm{COCH}_{2} \mathrm{CH}_{2} \mathrm{COOH}$

Fig. 1 Structures of compounds 1-28.

sitivity effect for further studies, the antiproliferative values should also be considered. The anticancer drugs etoposide and cisplatin were used as positive controls.

The antiproliferative activity and collateral sensitivity effect (RR values) of compounds $\mathbf{1 - 2 8}$ are summarized in $>$ Tables $\mathbf{1 - 3}$. As can be observed, a significant antiproliferative effect $\left(\mathrm{IC}_{50}\right.$ $<10 \mu \mathrm{M})$ in parental drug sensitive cell lines was observed for compounds 6 [EPG85-257, EPP85-181, and HT-29, IC $50=9.5 \mu \mathrm{M}$ $(\mathrm{Cl} 7.2-11.8 \mu \mathrm{M}), 7.1 \mu \mathrm{M}(\mathrm{Cl} 6.9-7.3 \mu \mathrm{M})$, and $6.7 \mu \mathrm{M}(\mathrm{Cl} 6.6-$ $6.8 \mu \mathrm{M})$, respectively], 7 [EPG85-257, $\mathrm{IC}_{50}=9.2 \mu \mathrm{M}(\mathrm{Cl} 8.0-$ $10.4 \mu \mathrm{M})$ ], 10 [EPG85-257, EPP85-181, IC $50=7.9 \mu \mathrm{M}(\mathrm{Cl} 7.4-$ $8.4 \mu \mathrm{M})$ and $6.7 \mu \mathrm{M}(\mathrm{Cl} 6.6-6.8 \mu \mathrm{M})$, respectively], 11 [HT-29, $\left.\mathrm{IC}_{50}=7.9 \mu \mathrm{M}(\mathrm{Cl} 4.1-11.4 \mu \mathrm{M})\right]$, and 15 [EPG85-257, $\mathrm{IC}_{50}=$ $8.0 \mu \mathrm{M}(\mathrm{Cl} 6.9-9.1 \mu \mathrm{M})]$. The remaining compounds showed a moderate/weak antiproliferative effect in parental drug-sensitive cell lines or were inactive ( $\bullet$ Tables $1-3$ ). Regarding MDR sublines, when the $\mathrm{IC}_{50}$ values were compared with those found for their specific drug-sensitive counterpart cell line, an increased sensitivity $(R R<1)$ was observed for most of the compounds ( $\vee$ Fig. 2), mainly for resistant cancer gastric and colon cancer cell lines. Moreover, a collateral sensitivity effect $(R R \leq 0.50)$ was observed for the natural compounds balsaminol $\mathrm{F}\left[3, \mathrm{IC}_{50}=6.2 \mu \mathrm{M}\right.$
$(\mathrm{Cl}$ 5.7-6.7 $\mu \mathrm{M}) ; \mathrm{RR}=0.43]$ and karavilagenin $\mathrm{C}\left[10, \mathrm{IC}_{50}=2.5 \mu \mathrm{M}\right.$ $(\mathrm{Cl} 2.2-2.8 \mu \mathrm{M}) ; \mathrm{RR}=0.32$ ] against the gastric EPG85-257 RDB subline ( $\vee$ Table 1 and Fig. 2 ), with a high concomitant antiproliferative activity, which was comparable to that found for the positive controls [cisplatin, $\mathrm{IC}_{50}=4.0 \mu \mathrm{M}(\mathrm{Cl} 3.7-4.3 \mu \mathrm{M})$; $\mathrm{RR}=1$; etoposide, $\mathrm{IC}_{50}=6.2 \mu \mathrm{M}(\mathrm{Cl} 5.9-6.5 \mu \mathrm{M}) ; \mathrm{RR}=59$ ]. A collateral sensitivity effect was also found for compounds 4 and 27 on the same cells, although with a lower antiproliferative effect. Similarly, on the gastric EPG85-257 RNOV variant, a collateral sensitivity effect was observed for compounds 3, 4, 6, and 9, and was associated with strong antiproliferative activity for compounds 3 [IC $\mathrm{I}_{50}=$ $7.2 \mu \mathrm{M}(\mathrm{Cl} 6.4-8.0 \mu \mathrm{M}) ; \mathrm{RR}=0.50]$ and $6\left[\mathrm{IC}_{50}=4.5 \mu \mathrm{M}(\mathrm{Cl} 2.5-\right.$ $6.5 \mu \mathrm{M}) ; \mathrm{RR}=0.47]$. By using the nonparametric Kruskal-Wallis rank test, a statistical difference with $p=0.053$ in the $I_{50}$ values was detected between the three gastric cell lines, and was more significant ( $p=0.010$, one tail) between EPG85-257 RDB and EPG85-257 RNOV cells.

Compounds $11\left[\mathrm{IC}_{50}=9.8 \mu \mathrm{M}(\mathrm{Cl} 7.4-12.2 \mu \mathrm{M}) ; \mathrm{RR}=0.51\right]$ and $3\left[\mathrm{IC}_{50}=8.5 \mu \mathrm{M}(\mathrm{Cl} 7.4-9.6 \mu \mathrm{M}) ; \mathrm{RR}=0.55\right]$ were the most selective against the pancreatic EPP85-181RDB cells. On the pancreatic EPP85-181RNOV subline, an RR $<1$ was also found for several compounds ( $\vee$ Table 2 and Fig. 2 ), indicating that they exerted a higher antiproliferative effect against the MDR-derived line than the parental one. Among them, compounds 3, 11, and 13 exhibited the lowest $\mathrm{IC}_{50}$ values $(11.7-13.7 \mu \mathrm{M})$. For the pancreatic cancer cell lines, a significant statistical difference was found between the $I C_{50}$ values $(p=0.03)$, reflecting a different antiproliferative effect of the compounds on both parental and resistant cancer cell lines. This effect was corroborated by the $p$ values obtained when $\mathrm{IC}_{50}$ values of the parental cell line and EPP85181RDB subline ( $p=0.016$, one tail) and $I_{50}$ values of EPP85181RDB and EPP85-181RNOV ( $p=0.009$, one tail) were compared.

Regarding colon cancer cell lines ( $\bullet$ Table 3 ), the best MDR-selective antiproliferative effects were found for karavilagenin $C[10$, $\mathrm{IC}_{50}=6.8 \mu \mathrm{M} \quad(\mathrm{Cl} \quad 5.2-8.4 \mu \mathrm{M}) ; \quad \mathrm{RR}=0.49, \quad \mathrm{EPG} 85-257 \quad \mathrm{RDB}$; $\mathrm{IC}_{50}=6.7 \mu \mathrm{M}(\mathrm{Cl} 5.8-7.6 \mu \mathrm{M}) ; \mathrm{RR}=0.49$, EPG85-257 RNOV] and some of its derivatives, with a collateral sensitivity effect being observed against both resistant variants. When comparing both the antiproliferative and the relative resistance ratio $(\vee$ Table 3 and Fig. 2), the best results were obtained for the acyl derivatives karavoate $\mathrm{A}$ [11, IC $\mathrm{I}_{50}=3.1 \mu \mathrm{M}(\mathrm{Cl} 1.8-4.4 \mu \mathrm{M})$; RR = 0.39, EPG85$257 \mathrm{RDB} ; \mathrm{IC}_{50}=2.3 \mu \mathrm{M}(\mathrm{Cl} 2.250-3.35 \mu \mathrm{M}) ; \mathrm{RR}=0.29$, EPG85-257 RNOV], karavoate $C\left[13, I_{50}=7.1 \mu \mathrm{M}(\mathrm{Cl} 7.09-7.10 \mu \mathrm{M})\right.$; $R R=$ 0.51, EPG85-257 RDB; IC $50=4.9 \mu \mathrm{M}(\mathrm{Cl} 4.3-5.5 \mu \mathrm{M}) ; \mathrm{RR}=0.36$, EPG85-257 RNOV], and karavoate $\mathrm{E}\left[15, \mathrm{IC}_{50}=6.9 \mu \mathrm{M}(\mathrm{Cl} 6.7-\right.$ $7.1 \mu \mathrm{M}) ; \quad R R=0.45$, EPG85-257 RDB; $\quad I_{50}=4.0 \mu \mathrm{M} \quad(\mathrm{Cl} 2.8-$ $5.2 \mu \mathrm{M}) ; \mathrm{RR}=0.26$, EPG85-257 RNOV].

When analyzing the results of compounds $1-28$, lipophilicity seems to be detrimental for antiproliferative activity, although no statistical correlation with antiproliferative activity was found. In fact, higher log p values ( $\geq 8.5$ ) (Table S1, Supporting Information), observed for the acyl derivatives, were always associated with a lack of antiproliferative effect $\left(\mathrm{IC}_{50}>100 \mu \mathrm{M}\right)$.

As mentioned before, these compounds (1-28) were previously assessed for their ability to modulate the transport activity of P-gp in a functional assay $[12,13]$. Interestingly, some compounds 
- Table 1 Antiproliferative activity of compounds 1-28 in gastric carcinoma cells: EPG85-257P (parental), EPG85-257RDB (MDR phenotype), and EPG85-257RNOV (MDR phenotype).

\begin{tabular}{|c|c|c|c|c|c|}
\hline \multirow[t]{2}{*}{ Compound } & \multirow{2}{*}{$\begin{array}{l}\text { EPG85-257P } \\
\text { IC }_{50}(\mu \mathrm{M})^{1} \\
(\mathrm{Cl} 95 \%)(\mu \mathrm{M})\end{array}$} & \multicolumn{2}{|l|}{ EPG85-257RDB } & \multicolumn{2}{|c|}{ EPG85-257RNOV } \\
\hline & & $\begin{array}{l}\mathrm{IC}_{50}(\mu \mathrm{M})^{1} \\
(\mathrm{Cl} 95 \%)(\mu \mathrm{M})\end{array}$ & $\mathbf{R R}^{2}$ & $\begin{array}{l}\mathrm{IC}_{50}(\mu \mathrm{M})^{1} \\
(\mathrm{Cl} 95 \%)(\mu \mathrm{M})\end{array}$ & $\mathbf{R R}^{2}$ \\
\hline Balsaminol A (1) & $\begin{array}{l}20.4 \\
(20.0-20.8)\end{array}$ & $\begin{array}{l}14.5 \\
(10.5-18.5)\end{array}$ & 0.71 & $\begin{array}{l}12.1 \\
(7.1-17.1)\end{array}$ & 0.59 \\
\hline Balsaminol D (2) & $>100$ & $\begin{array}{l}56.0 \\
(43.8-68.2)\end{array}$ & $<0.56$ & $\begin{array}{l}74.7 \\
(67.9-81.5)\end{array}$ & 0.75 \\
\hline Balsaminol F (3) & $\begin{array}{l}14.4 \\
(10.1-18.7)\end{array}$ & $\begin{array}{l}6.2 \\
(5.7-6.7)\end{array}$ & 0.43 & $\begin{array}{l}7.2 \\
(6.4-8.0)\end{array}$ & 0.50 \\
\hline Balsaminagenin $\mathrm{A}(4)$ & $\begin{array}{l}49.0 \\
(48.4-49.6)\end{array}$ & $\begin{array}{l}24.4 \\
(22.9-25.9)\end{array}$ & 0.50 & $\begin{array}{l}23.2 \\
(19.0-27.4)\end{array}$ & 0.47 \\
\hline Balsaminagenin B (5) & $\begin{array}{l}20.4 \\
(20.4-20.4)\end{array}$ & $\begin{array}{l}17.5 \\
(15.2-19.8)\end{array}$ & 0.86 & $\begin{array}{l}18.2 \\
(15.1-21.3)\end{array}$ & 0.89 \\
\hline Balsaminoside A (6) & $\begin{array}{l}9.5 \\
(7.2-11.8)\end{array}$ & $>100$ & $>10.52$ & $\begin{array}{l}4.5 \\
(2.5-6.5)\end{array}$ & 0.47 \\
\hline Balsaminoside B (7) & $\begin{array}{l}9.2 \\
(8.0-10.4)\end{array}$ & $\begin{array}{l}64.2 \\
(61.6-66.8)\end{array}$ & 6.98 & $\begin{array}{l}5.0 \\
(2.2-7.8)\end{array}$ & 0.54 \\
\hline Balsaminoside C (8) & $\begin{array}{l}19.8 \\
(19.1-20.5)\end{array}$ & $\begin{array}{l}58.8 \\
(49.8-67.8)\end{array}$ & 2.97 & $\begin{array}{l}11.5 \\
(4.8-18.2)\end{array}$ & 0.58 \\
\hline Cucurbalsaminol A (9) & $\begin{array}{l}67.0 \\
(64.1-69.9)\end{array}$ & $\begin{array}{l}54.7 \\
(46.4-63.0)\end{array}$ & 0.82 & $\begin{array}{l}31.9 \\
(24.0-39.8)\end{array}$ & 0.48 \\
\hline Karavilagenin C (10) & $\begin{array}{l}7.9 \\
(7.4-8.4)\end{array}$ & $\begin{array}{l}2.5 \\
(2.2-2.8)\end{array}$ & 0.32 & $\begin{array}{l}6.6 \\
(6.2-7.0)\end{array}$ & 0.84 \\
\hline Karavoate A (11) & $\begin{array}{l}19.8 \\
(19.4-20.2)\end{array}$ & $\begin{array}{l}13.1 \\
(10.7-15.5)\end{array}$ & 0.66 & $\begin{array}{l}16.8 \\
(12.2-21.4)\end{array}$ & 0.85 \\
\hline Karavoate B (12) & $\begin{array}{l}21.4 \\
(16.9-25.9)\end{array}$ & $\begin{array}{l}19.9 \\
(18.1-21.7)\end{array}$ & 0.93 & $\begin{array}{l}53.8 \\
(43.5-64.1)\end{array}$ & 2.51 \\
\hline Karavoate C (13) & $\begin{array}{l}19.3 \\
(18.6-20.0)\end{array}$ & $\begin{array}{l}21.2 \\
(19.4-23.0)\end{array}$ & 1.10 & $\begin{array}{l}10.4 \\
(7.8-13.0)\end{array}$ & 0.54 \\
\hline Karavoate D (14) & $\begin{array}{l}21.8 \\
(21.5-22.1)\end{array}$ & $>100$ & $>4.59$ & $\begin{array}{l}17.0 \\
(15.7-18.3)\end{array}$ & 0.78 \\
\hline Karavoate E (15) & $\begin{array}{l}8.0 \\
(6.9-9.1)\end{array}$ & $\begin{array}{l}63.5 \\
(60.9-66.1)\end{array}$ & 7.94 & $\begin{array}{l}7.0 \\
(6.7-7.3)\end{array}$ & 0.88 \\
\hline Karavoate G (17) & $\begin{array}{l}21.9 \\
(21.2-22.6)\end{array}$ & $>100$ & $>4.57$ & $\begin{array}{l}16.4 \\
(13.2-19.6)\end{array}$ & 0.75 \\
\hline Karavoate I (19) & $\begin{array}{l}19.6 \\
(19.0-20.2)\end{array}$ & $\begin{array}{l}74.1 \\
(66.0-82.2)\end{array}$ & 3.78 & $\begin{array}{l}14.3 \\
(13.5-15.1)\end{array}$ & 0.73 \\
\hline Karavoate K (21) & $\begin{array}{l}73.8 \\
(65.2-82.4)\end{array}$ & $>100$ & $>1.36$ & $\begin{array}{l}63.3 \\
(61.6-65.0)\end{array}$ & 0.86 \\
\hline Karavoate M (23) & $\begin{array}{l}20.0 \\
(19.2-20.8)\end{array}$ & $>100$ & $>5.00$ & $\begin{array}{l}17.9 \\
(15.2-20.6)\end{array}$ & 0.90 \\
\hline Karavoate O (25) & $\begin{array}{l}22.9 \\
(21.9-23.9)\end{array}$ & $>100$ & $>5.42$ & $\begin{array}{l}18.8 \\
(17.1-20.5)\end{array}$ & 0.82 \\
\hline Karavoate Q (27) & $\begin{array}{l}40.3 \\
(27.3-53.3)\end{array}$ & $\begin{array}{l}18.7 \\
(18.0-19.4)\end{array}$ & 0.46 & $\begin{array}{l}27.9 \\
(25.7-30.1)\end{array}$ & 0.69 \\
\hline Karavoate R (28) & $\begin{array}{l}66.6 \\
(66.0-67.2)\end{array}$ & $\begin{array}{l}55.7 \\
(54.9-56.5)\end{array}$ & 0.84 & $\begin{array}{l}62.9 \\
(59.0-66.8)\end{array}$ & 0.94 \\
\hline Etoposide & $\begin{array}{l}0.105 \\
(0.1-0.1)\end{array}$ & $\begin{array}{l}6.2 \\
(5.9-6.5)\end{array}$ & 59 & $\begin{array}{l}1.55 \\
(1.4-1.7)\end{array}$ & 14.8 \\
\hline Cisplatin & $\begin{array}{l}4.4 \\
(3.9-4.9)\end{array}$ & $\begin{array}{l}4.0 \\
(3.7-4.3)\end{array}$ & 1 & $\begin{array}{l}2.6 \\
(2.4-2.8)\end{array}$ & 0.6 \\
\hline
\end{tabular}

Compounds $16,18,20,22,24$, and 26 were ineffective in the sensitive and resistant variants of carcinoma cells $\left(\mathrm{IC}_{50}>100 \mu \mathrm{M}\right) .{ }^{1} \mathrm{The} \mathrm{IC} \mathrm{C}_{50}$ values with $95 \%$ confidence intervals ( $\mathrm{Cl} 95 \%$ ) given in parentheses indicate the mean of $\mathrm{n}=3$ to 4 independent experiments (each concentration was performed in triplicate per experiment). ${ }^{2} \mathrm{RR}$ is the relative resistance ratio determined by dividing the mean IC $\mathrm{C}_{50}$ against a resistant line by the mean $I C_{50}$ against a parental line 
- Table 2 Antiproliferative activity of compounds 1-28 in pancreatic carcinoma cells: EPP85-181P (parental), EPP85-181RDB (MDR phenotype), and EPP85-181RNOV (MDR phenotype).

\begin{tabular}{|c|c|c|c|c|c|}
\hline \multirow[t]{2}{*}{ Compounds } & \multirow{2}{*}{$\begin{array}{l}\text { EPP85-181P } \\
I_{50}(\mu \mathrm{M})^{1} \\
(\mathrm{Cl} 95 \%)(\mu \mathrm{M})\end{array}$} & \multicolumn{2}{|l|}{ EPP85-181RDB } & \multicolumn{2}{|l|}{ EPP85-181RNOV } \\
\hline & & $\begin{array}{l}I_{50}(\mu \mathrm{M})^{1}(\mathrm{Cl} \\
95 \%)(\mu \mathrm{M})\end{array}$ & $\mathbf{R R}^{2}$ & $\begin{array}{l}\mathrm{IC}_{50}(\mu \mathrm{M})^{1} \\
(\mathrm{Cl} 95 \%)(\mu \mathrm{M})\end{array}$ & $\mathbf{R R}^{2}$ \\
\hline Balsaminol A (1) & $\begin{array}{l}21.5 \\
(20.7-22.3)\end{array}$ & $\begin{array}{l}22.1 \\
(20.6-23.6)\end{array}$ & 1.03 & $\begin{array}{l}22.2 \\
(20.2-24.2)\end{array}$ & 1.03 \\
\hline Balsaminol D (2) & $\begin{array}{l}91.0 \\
(90.2-91.8)\end{array}$ & $>100$ & $>1.10$ & $>100$ & $>1.10$ \\
\hline Balsaminol F (3) & $\begin{array}{l}15.4 \\
(11.8-19.0)\end{array}$ & $\begin{array}{l}8.5 \\
(7.4-9.6)\end{array}$ & 0.55 & $\begin{array}{l}11.7 \\
(9.6-13.8)\end{array}$ & 0.76 \\
\hline Balsaminagenin A (4) & $\begin{array}{l}69.2 \\
(69.0-69.4)\end{array}$ & $\begin{array}{l}66.7 \\
(66.0-67.4)\end{array}$ & 0.96 & $\begin{array}{l}56.3 \\
(45.6-67.0)\end{array}$ & 0.81 \\
\hline Balsaminagenin B (5) & $\begin{array}{l}20.3 \\
(20.2-20.3)\end{array}$ & $\begin{array}{l}18.9 \\
(16.8-21.0)\end{array}$ & 0.93 & $\begin{array}{l}20.7 \\
(18.7-22.7)\end{array}$ & 1.02 \\
\hline Balsaminoside A (6) & $\begin{array}{l}7.1 \\
(6.9-7.3)\end{array}$ & $>100$ & $>14.08$ & $\begin{array}{l}9.6 \\
(9.4-9.8)\end{array}$ & 1.35 \\
\hline Balsaminoside B (7) & $\begin{array}{l}10.2 \\
(6.4-14.0)\end{array}$ & $\begin{array}{l}67.0 \\
(65.4-68.6)\end{array}$ & 6.57 & $\begin{array}{l}17.5 \\
(14.5-20.5)\end{array}$ & 1.72 \\
\hline Balsaminoside C (8) & $\begin{array}{l}21.0 \\
(20.4-21.6)\end{array}$ & $\begin{array}{l}66.0 \\
(64.9-67.1)\end{array}$ & 3.14 & $\begin{array}{l}20.1 \\
(19.6-20.6)\end{array}$ & 0.96 \\
\hline Cucurbalsaminol A (9) & $\begin{array}{l}69.0 \\
(66.8-71.2)\end{array}$ & $\begin{array}{l}70.0 \\
(68.8-71.2)\end{array}$ & 1.01 & $\begin{array}{l}68.5 \\
(67.6-69.4)\end{array}$ & 0.99 \\
\hline Karavilagenin C (10) & $\begin{array}{l}6.7 \\
(6.6-6.8)\end{array}$ & $\begin{array}{l}6.8 \\
(5.8-7.8)\end{array}$ & 1.00 & $\begin{array}{l}6.7 \\
(4.1-9.3)\end{array}$ & 1.00 \\
\hline Karavoate A (11) & $\begin{array}{l}19.1 \\
(17.4-20.8)\end{array}$ & $\begin{array}{l}9.8 \\
(7.4-12.2)\end{array}$ & 0.51 & $\begin{array}{l}13.4 \\
(10.4-16.4)\end{array}$ & 0.70 \\
\hline Karavoate B (12) & $\begin{array}{l}55.1 \\
(48.4-61.8)\end{array}$ & $\begin{array}{l}85.6 \\
(81.6-89.6)\end{array}$ & 1.55 & $\begin{array}{l}33.6 \\
(25.9-41.3)\end{array}$ & 0.61 \\
\hline Karavoate C (13) & $\begin{array}{l}19.7 \\
(19.4-20.0)\end{array}$ & $\begin{array}{l}23.9 \\
(23.0-24.8)\end{array}$ & 1.21 & $\begin{array}{l}13.7 \\
(8.4-19.0)\end{array}$ & 0.70 \\
\hline Karavoate D (14) & $\begin{array}{l}29.0 \\
(22.1-35.9)\end{array}$ & $>100$ & $>3.45$ & $\begin{array}{l}20.8 \\
(20.78-20.82)\end{array}$ & 0.72 \\
\hline Karavoate E (15) & $\begin{array}{l}19.4 \\
(18.7-20.1)\end{array}$ & $\begin{array}{l}77.1 \\
(73.6-80.6)\end{array}$ & 3.97 & $\begin{array}{l}14.9 \\
(12.6-17.2)\end{array}$ & 0.77 \\
\hline Karavoate G (17) & $\begin{array}{l}62.1 \\
(47.6-76.6)\end{array}$ & $>100$ & $>1.61$ & $\begin{array}{l}63.7 \\
(44.3-83.1)\end{array}$ & 1.03 \\
\hline Karavoate I (19) & $\begin{array}{l}23.9 \\
(23.6-24.2)\end{array}$ & $>100$ & $>4.18$ & $\begin{array}{l}20.6 \\
(20.4-20.8)\end{array}$ & 0.86 \\
\hline Karavoate K (21) & $>100$ & $>100$ & n.d. & $\begin{array}{l}66.8 \\
(65.6-68.0)\end{array}$ & $<0.67$ \\
\hline Karavoate M (23) & $\begin{array}{l}23.7 \\
(23.1-24.3)\end{array}$ & $>100$ & $>4.22$ & $\begin{array}{l}20.4 \\
(20.2-20.6)\end{array}$ & 0.86 \\
\hline Karavoate O (25) & $\begin{array}{l}28.2 \\
(27.7-28.7)\end{array}$ & $>100$ & $>3.55$ & $\begin{array}{l}22.5 \\
(20.8-24.2)\end{array}$ & 0.80 \\
\hline Karavoate Q (27) & $\begin{array}{l}49.1 \\
(41.1-57.1)\end{array}$ & $\begin{array}{l}58.6 \\
(55.2-62.0)\end{array}$ & 1.19 & $\begin{array}{l}55.5 \\
(54.9-56.1)\end{array}$ & 1.13 \\
\hline Karavoate R (28) & $\begin{array}{l}68.6 \\
(68.1-69.1)\end{array}$ & $\begin{array}{l}70.3 \\
(68.4-72.2)\end{array}$ & 1.02 & $\begin{array}{l}71.4 \\
(70.4-72.4)\end{array}$ & 1.04 \\
\hline Etoposide & $\begin{array}{l}0.58 \\
(0.57-0.59)\end{array}$ & $\begin{array}{l}62.0 \\
(57.2-66.8)\end{array}$ & 106.9 & $\begin{array}{l}4.5 \\
(3.7-5.3)\end{array}$ & 7.8 \\
\hline Cisplatin & $\begin{array}{l}0.08 \\
(0.07-0.09)\end{array}$ & $\begin{array}{l}0.09 \\
(0.07-0.1)\end{array}$ & 1.2 & $\begin{array}{l}2.6 \\
(2.4-2.8)\end{array}$ & 34 \\
\hline
\end{tabular}

Compounds $16,18,20,22,24$, and 26 were ineffective in the sensitive and resistant variants of carcinoma cells $\left(\mathrm{IC}_{50}>100 \mu \mathrm{M}\right) .{ }^{1} \mathrm{The} \mathrm{IC}_{50}$ values with $95 \%$ confidence intervals ( $\mathrm{Cl} 95 \%$ ) given in parentheses indicate the mean of $\mathrm{n}=3$ to 4 independent experiments (each concentration was performed in triplicate per experiment). ${ }^{2} \mathrm{RR}$ is the relative resistance ratio determined by dividing the mean $\mathrm{IC}_{50}$ against a resistant line by the mean $I C_{50}$ against a parental line 
- Table 3 Antiproliferative activity of compounds 1-28 in colon carcinoma cells: HT-29P (parental), HT-29RDB (MDR phenotype), and HT-29RNOV (MDR phenotype).

\begin{tabular}{|c|c|c|c|c|c|}
\hline \multirow[t]{2}{*}{ Compounds } & \multirow{2}{*}{$\begin{array}{l}\text { HT-29P } \\
\begin{array}{l}\mathrm{IC}_{50}(\mu \mathrm{M})^{1} \\
(\mathrm{Cl} 95 \%)(\mu \mathrm{M})\end{array}\end{array}$} & \multicolumn{2}{|l|}{ HT-29RDB } & \multicolumn{2}{|l|}{ HT-29RNOV } \\
\hline & & $\begin{array}{l}\mathrm{IC}_{50}(\mu \mathrm{M})^{1} \\
(\mathrm{Cl} 95 \%)(\mu \mathrm{M})\end{array}$ & $\mathbf{R R}^{2}$ & $\begin{array}{l}\mathrm{IC}_{50}(\mu \mathrm{M})^{1} \\
(\mathrm{Cl} 95 \%)(\mu \mathrm{M})\end{array}$ & $\mathbf{R R}^{2}$ \\
\hline Balsaminol A (1) & $\begin{array}{l}21.2 \\
(21.0-21.4)\end{array}$ & $\begin{array}{l}21.0 \\
(20.4-21.6)\end{array}$ & 0.99 & $\begin{array}{l}17.5 \\
(16.9-18.1)\end{array}$ & 0.83 \\
\hline Balsaminol D (2) & $>100$ & $\begin{array}{l}79.0 \\
(73.0-85.0)\end{array}$ & $<0.79$ & $\begin{array}{l}79.0 \\
(72.1-85.9)\end{array}$ & $<0.79$ \\
\hline Balsaminol F (3) & $\begin{array}{l}11.9 \\
(11.1-12.6)\end{array}$ & $\begin{array}{l}9.2 \\
(7.5-10.9)\end{array}$ & 0.77 & $\begin{array}{l}7.0 \\
(6.7-7.3)\end{array}$ & 0.59 \\
\hline Balsaminagenin A (4) & $\begin{array}{l}60.4 \\
(59.9-60.9)\end{array}$ & $\begin{array}{l}57.3 \\
(49.0-65.6)\end{array}$ & 0.95 & $\begin{array}{l}31.3 \\
(29.9-32.7)\end{array}$ & 0.52 \\
\hline Balsaminagenin B (5) & $\begin{array}{l}20.1 \\
(20.0-20.2)\end{array}$ & $\begin{array}{l}20.0 \\
(19.5-20.5)\end{array}$ & 1.00 & $\begin{array}{l}19.1 \\
(18.3-19.9)\end{array}$ & 0.95 \\
\hline Balsaminoside A (6) & $\begin{array}{l}6.7 \\
(6.6-6.8)\end{array}$ & $\begin{array}{l}7.1 \\
(6.5-7.7)\end{array}$ & 1.06 & $\begin{array}{l}4.8 \\
(2.6-7.0)\end{array}$ & 0.72 \\
\hline Balsaminoside B (7) & $\begin{array}{l}18.2 \\
(16.0-20.4)\end{array}$ & $\begin{array}{l}61.7 \\
(57.5-65.9)\end{array}$ & 3.39 & $\begin{array}{l}15.9 \\
(14.9-16.9)\end{array}$ & 0.87 \\
\hline Balsaminoside C (8) & $\begin{array}{l}27.8 \\
(27.0-28.6)\end{array}$ & $\begin{array}{l}66.4 \\
(66.2-66.6)\end{array}$ & 2.39 & $\begin{array}{l}31.4 \\
(26.3-36.5)\end{array}$ & 1.13 \\
\hline Cucurbalsaminol A (9) & $\begin{array}{l}66.4 \\
(64.8-68.0)\end{array}$ & $\begin{array}{l}55.9 \\
(49.0-62.8)\end{array}$ & 0.84 & $\begin{array}{l}48.9 \\
(39.5-58.3)\end{array}$ & 0.74 \\
\hline Karavilagenin C (10) & $\begin{array}{l}13.8 \\
(13.1-14.5)\end{array}$ & $\begin{array}{l}6.8 \\
(5.2-8.4)\end{array}$ & 0.49 & $\begin{array}{l}6.7 \\
(5.8-7.6)\end{array}$ & 0.49 \\
\hline Karavoate A (11) & $\begin{array}{l}7.9 \\
(4.1-11.4)\end{array}$ & $\begin{array}{l}3.1 \\
(1.8-4.4)\end{array}$ & 0.39 & $\begin{array}{l}2.3 \\
(2.25-3.35)\end{array}$ & 0.29 \\
\hline Karavoate B (12) & $\begin{array}{l}61.5 \\
(49.4-73.6)\end{array}$ & $\begin{array}{l}30.7 \\
(8.5-52.9)\end{array}$ & 0.50 & $\begin{array}{l}19.3 \\
(12.3-26.3)\end{array}$ & 0.31 \\
\hline Karavoate C (13) & $\begin{array}{l}13.8 \\
(10.7-16.9)\end{array}$ & $\begin{array}{l}7.1 \\
(7.09-7.1)\end{array}$ & 0.51 & $\begin{array}{l}4.9 \\
(4.3-5.5)\end{array}$ & 0.36 \\
\hline Karavoate E (15) & $\begin{array}{l}15.4 \\
(14.9-15.9)\end{array}$ & $\begin{array}{l}6.9 \\
(6.7-7.1)\end{array}$ & 0.45 & $\begin{array}{l}4.0 \\
(2.8-5.2)\end{array}$ & 0.26 \\
\hline Karavoate G (17) & $\begin{array}{l}80.1 \\
(77.2-83.0)\end{array}$ & $\begin{array}{l}67.1 \\
(52.6-81.6)\end{array}$ & 0.83 & $\begin{array}{l}27.5 \\
(25.3-29.7)\end{array}$ & 0.34 \\
\hline Karavoate I (19) & $\begin{array}{l}27.7 \\
(26.5-28.9)\end{array}$ & $\begin{array}{l}22.5 \\
(21.6-23.4)\end{array}$ & 0.81 & $\begin{array}{l}22.3 \\
(20.5-24.1)\end{array}$ & 0.81 \\
\hline Karavoate M (23) & $\begin{array}{l}28.7 \\
(27.8-29.6)\end{array}$ & $\begin{array}{l}14.7 \\
(9.4-20.0)\end{array}$ & 0.39 & $\begin{array}{l}18.9 \\
(17.9-19.9)\end{array}$ & 0.66 \\
\hline Karavoate O (25) & $\begin{array}{l}71.3 \\
(68.0-74.6)\end{array}$ & $\begin{array}{l}27.9 \\
(19.9-35.9)\end{array}$ & 0.39 & $\begin{array}{l}25.9 \\
(22.5-29.3)\end{array}$ & 0.36 \\
\hline Karavoate Q (27) & $\begin{array}{l}61.3 \\
(59.1-63.5)\end{array}$ & $\begin{array}{l}24.2 \\
23.8-24.6)\end{array}$ & 0.39 & $\begin{array}{l}21.4 \\
(21.2-21.6)\end{array}$ & 0.34 \\
\hline Karavoate R (28) & $\begin{array}{l}69.4 \\
(68.2-70.6)\end{array}$ & $\begin{array}{l}67.4 \\
(66.7-68.1)\end{array}$ & 0.97 & $\begin{array}{l}62.2 \\
(57.4-67.0)\end{array}$ & 0.90 \\
\hline Etoposide & $\begin{array}{l}2.3 \\
(2.0-2.6)\end{array}$ & $\begin{array}{l}26.0 \\
(24.1-27.9)\end{array}$ & 11.3 & $\begin{array}{l}35.0 \\
(32.1-37.9)\end{array}$ & 15.2 \\
\hline Cisplatin & $\begin{array}{l}3.8 \\
(3.7-3.9)\end{array}$ & $\begin{array}{l}2.7 \\
(2.6-2.8)\end{array}$ & 0.7 & $\begin{array}{l}3.8 \\
(3.7-3.9)\end{array}$ & 1 \\
\hline
\end{tabular}

Compounds $14,16,18,20-22,24$, and 26 were ineffective in the sensitive and resistant variants of carcinoma cells $\left(I_{50}>100 \mu M\right) .{ }^{1}$ The $I C_{50}$ values with $95 \%$ confidence intervals ( $\mathrm{Cl} 95 \%$ ) given in parentheses indicate the mean of $\mathrm{n}=3$ to 4 independent experiments (each concentration was performed in triplicate per experiment). ${ }^{2} \mathrm{RR}$ is the (elative resistance ratio determined by dividing the mean $\mathrm{IC}_{50}$ against a resistant line by the mean $\mathrm{IC}_{50}$ against a parental line. 


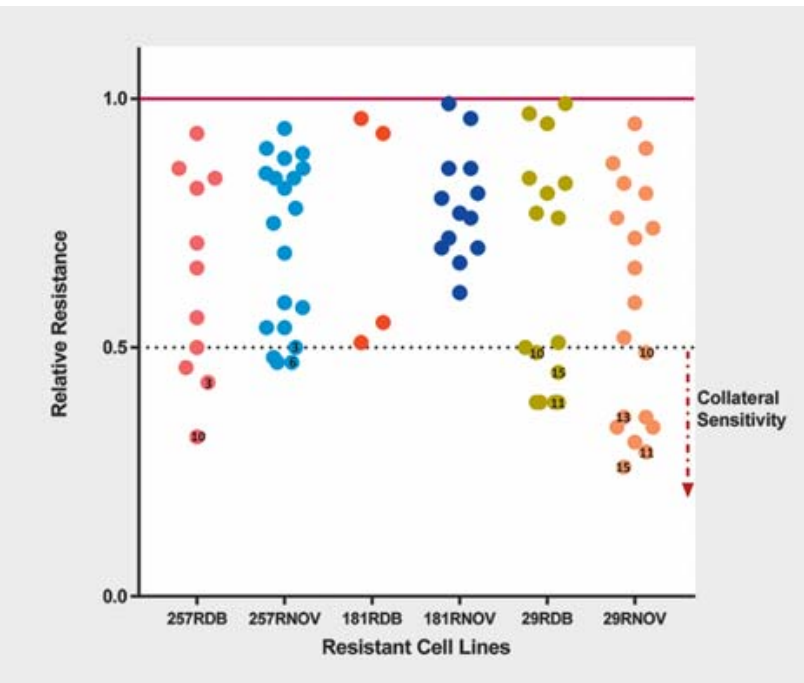

- Fig. 2 Compounds that showed greater sensitivity to the MDR sublines than to the corresponding parental cell line: relative resistance, the ratio between the mean $\mathrm{IC}_{50}$ against a resistant line by the mean $\mathrm{IC}_{50}$ against a parental line (RR), lower than 1.0. Compounds $4,6,9-13,15,17,23,25$, and 27 exhibited a collateral effect $(R R \leq 0.50)$. The tagged relative resistance points correspond to compounds that presented the best collateral sensitivity effect values concomitant with significant antiproliferative activity.

classified as strong P-gp modulators $(1,4-7,10-13,15)$ also showed a significant collateral sensitivity effect on some of the resistant cell sublines $[12,13]$. For instance, karavilagenin $C(10)$, which presented a very strong P-gp-mediated MDR reversal activity at a very low concentration [12], was able to kill the resistant gastric cell line EPG85 (RR $=0.32)$ more efficiently. Although to a lesser extent, a selective antiproliferative effect was also observed against both resistant colon HT-29 cell sublines ( $R R<0.50)$. On the other hand, the monoacetylated derivative of 10, karavoate $A(11)$, which was also able to modulate P-gp [12], exhibited a collateral sensitivity [16] effect against both resistant HT-29 colon carcinoma cell variants (HT-29RDB, RR = 0.39; HT-29RNOV, RR=0.29). As expected, similar results were also found for the efflux pump modulator karavoate $E$ (15), which differs from compound 11 in the number of carbons of the ester moiety. Similarly, a collateral sensitivity effect $(R R=0.47)$ was also observed for the strong P-gp modulator 6 [12] against EPG85-257RNOV gastric cancer cells.

The exact molecular mechanisms mediating a sensitization of different multidrug-resistant cancer cell variants to alternative triterpenoids have still not been evaluated and are beyond the scope of this investigation.

In conclusion, MDR is a complex phenomenon, involving several biochemical mechanisms. Thus, some of these triterpenes, such as compounds $6,10,11$, and 15 , by acting as both P-gp modulators and collateral sensitivity agents, might be promising leads for overcoming MDR cancer cells and are worthy of further studies.

\section{Materials and Methods}

\section{Tested compounds}

Compounds 1-10, namely, balsaminol A (1), balsaminol D (2), balsaminol $F(3)$, balsaminagenin A (4), balsaminagenin B (5), balsaminosides $A-C(6-8)$, cucurbalsaminol $A(9)$, and karavilagenin $C(10)$, were previously isolated from the methanol extract of M. balsamina as reported [12,21-23]. Compound 10 , isolated in a large amount, gave rise to 18 compounds, namely, karavoates A-R (11-28), by using several alkanoyl and aroyl acylating reagents, as described $[12,24]$. The purity of the compounds was more than $95 \%$ by HPLC. All of the compounds were dissolved in DMSO.

\section{Cell lines and cell culture}

The human cancer cell lines (EPG85-257P, EPP85-181P, and HT29P) and their drug-resistant sublines (EPG85-257RNOV, EPG85257RDB, EPP85-181RNOV, EPP85-181RDB, HT-29RNOV, and HT29RDB) were grown in Leibovitz L-15 medium (Biowhittaker) supplemented with $10 \%$ fetal calf serum (GIBCO/BRL), $1 \mathrm{mM}$ L-glutamine, $6.25 \mathrm{mg} / \mathrm{L}$ fetuin, $80 \mathrm{IE} / \mathrm{L}$ insulin, $2.5 \mathrm{mg} / \mathrm{mL}$ transferrin, $0.5 \mathrm{~g} / \mathrm{L}$ glucose, $1.1 \mathrm{~g} / \mathrm{L} \mathrm{NaHCO}_{3}, 1 \%$ minimal essential vitamins, and $20000 \mathrm{kIE} / \mathrm{L}$ trasylolina in a humidified atmosphere of $5 \%$ $\mathrm{CO}_{2}$ at $37^{\circ} \mathrm{C}$. The drug-resistant cell lines were established from parental cell lines by continuous exposure of the cells to stepwise increasing concentrations of antineoplastic agents as described previously [29]. For maintenance of drug-resistant phenotypes, the medium of the drug-resistant sublines was supplemented with the selective agent as described previously [30]. The used cytotoxic drugs daunorubicin (Farmitalia Carlo Erba), mitoxantrone (Lederle), etoposide (Bristol-Myers), and cisplatin (GRY-Pharm) showed purities for application in clinical settings.

\section{Cell proliferation assay}

The antiproliferative activity of the compounds was assessed using a proliferation assay based on SRB staining as described previously [17]. Briefly, 800 cells per well were seeded in 96-well plates in triplicate. After $24 \mathrm{~h}$ attachment, the particular agent was added in a dilution series for 5 days incubation $\left(5 \% \mathrm{CO}_{2}\right.$ at $37^{\circ} \mathrm{C}$ ). Cells were fixed by chilled $10 \%$ trichloroacetic acid for $1 \mathrm{~h}$ at $4{ }^{\circ} \mathrm{C}$, and washed five times with tap water before staining was performed with $0.4 \%$ SRB in $1 \%$ acetic acid for $10 \mathrm{~min}$ at room temperature. After washing with $1 \%$ acetic acid, drying, and resolubilization in $20 \mathrm{mM}$ Tris- $\mathrm{HCl}(\mathrm{pH} 10)$, absorbance was measured at $562 \mathrm{~nm}$ against the reference wavelength of $690 \mathrm{~nm}$. Etoposide and cisplatin were used as positive controls. Mean $\mathrm{IC}_{50}$ values with a $95 \%$ confidence interval were calculated from four independent experiments in triplicate for each cell line by using Prism software (GraphPad Software, Inc.). RR values were determined as $I C_{50 \text { (resistant cells) } /} / \mathrm{C}_{50 \text { (parental cells). }}$.

\section{Statistical analysis}

Analysis using the nonparametric Kruskal-Wallis rank test (a probability value of $p<0.05$ was considered statistically significant) was carried out to identify differences between the three cells lines of each group. The Mann-Whitney test was used to examine 
the statistical significance (a probability value of $p<0.05$ was considered statistically significant) of differences in the mean $\mathrm{IC}_{50}$ values between two independent groups. The Real Stats package of Excel software was used.

\section{Supporting information}

Physicochemical properties of compounds $\mathbf{1 - 2 8}$ are available as Supporting Information.

\section{Acknowledgements}

This study was financially supported by European Structural \& Investment Funds through the COMPETE Programme and from National Funds through FCT - Fundação para a Ciência e a Tecnologia- under the Programme grant SAICTPAC/0019/2015 and project PTDC/MED-QUI/ 30591/2017. The authors thank the Portuguese Embassy in Mozambique as well as the Portuguese Office of International Affairs for plant transport.

\section{Conflict of Interest}

The authors declare no conflict of interest.

\section{References}

[1] Szakács G, Hall MD, Gottesman MM, Boumendjel A, Kachadourian R, Day BJ, Baubichon-Cortay H, Di Pietro A. Targeting the Achilles heel of multidrug-resistant cancer by exploiting the fitness cost of resistance. Chem Rev 2014; 114: 5753-5774

[2] Gottesman MM, Lavi O, Hall MD, Gillet JP. Toward a better understanding of the complexity of cancer drug resistance. Annu Rev Pharmacol Toxicol 2016; 56: 85-102

[3] Lagas JS, van Waterschoot RAB, Sparidans RW, Wagenaar E, Beijnen JH, Schinkel AH. Breast cancer resistance protein and P-glycoprotein limit sorafenib brain accumulation. Mol Cancer Ther 2010; 9: 319-326

[4] Marchetti S, de Vries NA, Buckle T, Bolijn M], van Eijndhoven MAJ, Beijnen JH, Mazzanti R, van Tellingen O, Schellens JHM. Effect of the ATP-binding cassette drug transporters $A B C B 1, A B C G 2$, and $A B C C 2$ on erlotinib hydrochloride (Tarceva) disposition in in vitro and in vivo pharmacokinetic studies employing Bcrp1-/-/Mdr1a/1b-/- (triple-knockout) and wild-type mice. Mol Cancer Ther 2008; 7: 2280-2287

[5] Ozvegy-Laczka C, Cserepes ], Elkind NB, Sarkadi B. Tyrosine kinase inhibitor resistance in cancer: role of $A B C$ multidrug transporters. Drug Resist Updat 2005; 8: 15-26

[6] Saraswathy M, Gong S. Different strategies to overcome multidrug resistance in cancer. Biotechnol Adv 2013; 31: 1397-1407

[7] Klukovits A, Krajcsi P. Mechanisms and therapeutic potential of inhibiting drug efflux transporters. Expert Opin Drug Metab Toxicol 2015; 11: 907-920

[8] Pluchino KM, Hall MD, Goldsborough AS, Callaghan R, Gottesman MM Collateral sensitivity as a strategy against cancer multidrug resistance. Drug Resist Updat 2012; 15: 98-105

[9] Thakur GS, Bag M, Sanodiya BS, Bhadouriya P, Debnath M, Prasad GB, Bisen PS. Momordica balsamina: a medicinal and neutraceutical plant for health care management. Curr Pharm Biotechnol 2009; 10: 667-682

[10] Chen JC, Chiu MH, Nie RL, Cordell GA, Qiu SX. Cucurbitacins and cucurbitane glycosides: structures and biological activities. Nat Prod Rep 2005; 22: 386-399

[11] Dandawate PR, Subramaniam D, Padhye SB, Anant S. Bitter melon: a panacea for inflammation and cancer. Chin J Nat Med 2016; 14: 81-100
[12] Ramalhete C, Molnár ], Mulhovo S, Rosário VE, Ferreira MJU. New potent P-glycoprotein modulators with the cucurbitane scaffold and their synergistic interaction with doxorubicin on resistant cancer cells. Bioorg Med Chem 2009; 17: 6942-6951

[13] Ramalhete C, Mulhovo S, Molnar J, Ferreira MJU. Triterpenoids from Momordica balsamina: Reversal of ABCB1-mediated multidrug resistance. Bioorg Med Chem 2016; 24: 5061-5067

[14] Ramalhete C, Spengler G, Martins A, Martins M, Viveiros M, Mulhovo S, Ferreira MJU, Amaral L. Inhibition of efflux pumps in methicillin-resistant Staphylococcus aureus and Enterococcus faecalis resistant strains by triterpenoids from Momordica balsamina. Int ] Antimicrob Agents 2011; 37: 70-74

[15] Reis MA, Ahmed OB, Spengler G, Molnár ], Lage H, Ferreira MJU. Exploring jolkinol $\mathrm{D}$ derivatives to overcome multidrug resistance in cancer. J Nat Prod 2017; 80: 1411-1420

[16] Reis MA, Ahmed OB, Spengler G, Molnár J, Lage H, Ferreira MJU. Jatrophane diterpenes and cancer multidrug resistance - $A B C B 1$ efflux modulation and selective cell death induction. Phytomedicine 2016; 23: 968-978

[17] Reis MA, Paterna A, Ferreira RJ, Lage H, Ferreira MJU. Macrocyclic diterpenes resensitizing multidrug resistant phenotypes. Bioorg Med Chem 2014; 22: 3696-3702

[18] Paterna A, Kincses A, Spengler G, Mulhovo S, Molnár J, Ferreira MJU. Dregamine and tabernaemontanine derivatives as $A B C B 1$ modulators on resistant cancer cells. Eur J Med Chem 2017; 128: 247-257

[19] Ferreira MJU, Duarte N, Reis M, Madureira AM, Molnár J. Euphorbia and Momordica metabolites for overcoming multidrug resistance. Phytochem Rev 2014; 13: 915-935

[20] Matos AM, Reis M, Duarte N, Spengler G, Molnár ], Ferreira MJU. Epoxylathyrol derivatives: modulation of $A B C B 1$-mediated multidrug resistance in human colon adenocarcinoma and mouse T-lymphoma cells. J Nat Prod 2015; 78: 2215-2228

[21] Ramalhete C, da Cruz FP, Lopes D, Mulhovo S, Rosário VE, Prudêncio M, Ferreira MJU. Triterpenoids as inhibitors of erythrocytic and liver stages of Plasmodium infections. Bioorg Med Chem 2011; 19: 7474-7481

[22] Ramalhete C, Lopes D, Mulhovo S, Molnár J, Rosário VE, Ferreira MJU. New antimalarials with a triterpenic scaffold from Momordica balsamina. Bioorg Med Chem 2010; 18: 5254-5260

[23] Ramalhete C, Mansoor TA, Mulhovo S, Molnár J, Ferreira MJU. Cucurbitane-type triterpenoids from the African plant Momordica balsamina. J Nat Prod 2009; 72: 2009-2013

[24] Ramalhete C, Lopes D, Molnár ], Mulhovo S, Rosário VE, Ferreira MJU. Karavilagenin C derivatives as antimalarials. Bioorg Med Chem 2011; 19: $330-338$

[25] Duarte N, Lage H, Abrantes M, Ferreira MJU. Phenolic compounds as selective antineoplasic agents against multidrug-resistant human cancer cells. Planta Med 2010; 76: 975-980

[26] Lage H, Duarte N, Coburger C, Hilgeroth A, Ferreira MJU. Antitumor activity of terpenoids against classical and atypical multidrug resistant cancer cells. Phytomedicine 2010; 17: 441-448

[27] Hilgeroth A, Baumert C, Coburger C, Seifert M, Krawczyk S, Hempel C, Neubauer F, Krug M, Molnár J, Lage $\mathrm{H}$. Novel structurally varied $\mathrm{N}$-alkyl 1,4-dihydropyridines as $A B C B 1$ inhibitors: structure-activity relationships, biological activity and first bioanalytical evaluation. Med Chem 2013; 9: 487-493

[28] Hall MD, Handley MD, Gottesman MM. Is resistance useless? Multidrug resistance and collateral sensitivity. Trends Pharmacol Sci 2009; 30: 546-556

[29] Lage H. Proteomic approaches for investigation of therapy resistance in cancer. Proteomics. Clin Appl 2009; 3: 883-911

[30] Lage H, Aki-Sener E, Yalcin I. High antineoplastic activity of new heterocyclic compounds in cancer cells with resistance against classical DNA topoisomerase II-targeting drug. Int ] Cancer 2006; 119: 213-220 